Acute exercise effects on smoking withdrawal symptoms and desire to smoke are not related to expectation

Pages: 22

Word count: 2487

James Z Daniel ${ }^{1}$, Mark Cropley ${ }^{2}$ \& Chris Fife-Schaw ${ }^{2}$

${ }^{1}$ Department of Psychology, University of the West of England, Coldharbour Lane,

Bristol BS16 1QY, UK ${ }^{2}$ Department of Psychology, University of Surrey, Guildford, Surrey, GU2 7XH, UK

Correspondence to:

Dr James Daniel

Department of Psychology

Faculty of Applied Sciences

University of the West of England

Coldharbour Lane, Bristol

BS16 1QY, UK

Tel: 01173283975

Fax: 01173282904

E-mail: James.Daniel@uwe.ac.uk 


\title{
Acute exercise effects on smoking withdrawal symptoms and desire to smoke are not related to expectation
}

\author{
J Z Daniel, M Cropley and C Fife-Schaw
}

\begin{abstract}
Rationale Recent research has shown that 10 minutes of moderate intensity exercise reduce smoking withdrawal symptoms and desire to smoke in acutely abstinent smokers. The aim of the current study was to determine whether the reductions are related to participant expectation of these effects.

Method Forty-five sedentary participants who had smoked 10 or more cigarettes per day for at least three years reported their expectation of the effects of exercise on smoking withdrawal symptoms. Approximately one month later participants were randomly assigned to one of three groups after 11-15 hours of overnight smoking abstinence. Each group read either a positive, negative or neutral statement concerning exercise effects on smoking withdrawal symptoms. They rated their expectation again and then completed 10 minutes of moderate intensity exercise on a stationary bicycle ergometer. Using standardised scales, participants rated smoking withdrawal symptoms and desire to smoke at 10,5 , and 0 minutes before exercise, then at 5 and 10 minutes during exercise and 15 and 20 minutes post exercise.

Results Expectation of exercise effects on withdrawal were manipulated in the predicted directions. No significant group main effects were found for any symptom. Significant reductions in symptoms and desire to smoke occurred during and after exercise regardless of participant expectation.
\end{abstract}


Conclusions Ten minutes of moderate intensity exercise can lead to reductions in desire to smoke and smoking withdrawal symptoms which is not due to participant's expectation of exercise effects. These findings support the use of short periods of exercise as an aid to smoking cessation.

KEYWORDS smoking cessation, exercise, expectation, withdrawal 


\section{INTRODUCTION}

The positive effects of moderate intensity exercise on acute smoking withdrawal symptoms and desire to smoke have now been demonstrated in a number of studies (Daniel et al. 2004; Taylor et al. 2005; Taylor et al. 2007; Ussher et al. 2001). Although previous research suggests that distraction is not responsible for changes in smoking withdrawal symptoms following exercise (Daniel et al. 2004; Daniel et al. 2006), there are a number of other psychological factors that may be involved in this phenomenon. No published research investigating exercise effects on cravings has explicitly attempted to manipulate expectancy in order to determine its effects on smoking related outcomes. Expectation of the effects of exercise on withdrawal may be related to the actual reduction in desire for a cigarette and smoking withdrawal symptoms following 10 minutes of moderate intensity exercise.

It has been proposed that expectancy of the psychological benefits of exercise on mood may affect actual changes in mood associated with physical activity (Salmon 2001). Expectation of this effect might develop in several ways. For example, media reports of the positive effects exercise has on mood are a possible source of expectancy, as are personal experiences and anecdotal reports. Desharnais et al (1993) compared two groups of participants that took part in a ten-week exercise programme, and one group was provided with information about the psychological benefits of exercise. This group reported significantly higher self-esteem and reported better psychological well-being in the middle and at the end of the ten-week exercise programme compared with the control group, who received no additional information about exercise effects on mood. Pierce et al (1993) examined chronic changes in psychological and cognitive variables associated with participation in aerobic, 
strength and flexibility training programmes. Expectancy of the effects of exercise was measured using self-report instruments. Findings showed that significantly greater changes in concentration, mood and energy level reflected participant's expectancies of improvement. It is possible that the effect of expectation may also be similar in expectation of specific mood effects, such as mood states associated with smoking withdrawal. These findings have been supported elsewhere (O'Halloran et al. 2002). Other studies, however, have refuted the role of expectation in exercise and mood (Berger et al. 1998; Youngstedt et al. 1993) although these studies suffer from a number of methodological limitations, i.e. validity of the measures used, nonrepresentative sample, lack of applicability to acute exercise interventions.

Expectancy can be based on actual experiences, previous reactions to similar situations or based on knowledge of how others have reacted (Wilson et al. 1989). According to this possibility an individual who has a strong belief that exercise will reduce smoking withdrawal symptoms and desire to smoke will experience greater reductions compared with someone who does not have this expectation, or has a weaker expectation of the effect.

It has also been suggested that judgements people make are influenced by the relative availability of information, or accessibility from memory of events related to the judgement they are about to make (Schwarz 2002; Williams et al. 1997). It may therefore be possible, by providing information to participants prior to making judgements, to influence their expectation of the effects exercise will have on smoking withdrawal mood states and desire to smoke. Utilising this approach this study will investigate whether expectation of exercise is related to reductions in 
cigarette cravings and desire to smoke in acutely abstinent smokers following a short bout of moderate intensity exercise.

\section{METHODS}

\section{$\underline{\text { Sample }}$}

Forty five smokers ( $n=22$ male, 23 female) were recruited via advertisements placed around the University of Surrey campus. Participants were between 16 and 65 years of age, not receiving any form of psychiatric treatment, smoking at least 10 or more cigarettes a day for at least three years and self-reported to be sedentary. Being sedentary was defined as not engaging in vigorous exercise three or more times a week for at least 20 minutes each time, or doing some moderate intensity activity at least five times a week for 30 minute periods or more (Franklin 2000). Full ethical approval for the study was granted via the University of Surrey Advisory Committee on Ethics.

\section{Procedure and materials}

Initially (time 1) participants completed 8 questions of the physical activity readiness questionnaire (PAR-Q) to screen for exercise eligibility (Thomas et al. 1992). At this point participants' carbon monoxide (CO) was measured using a Bedfont smokerlyzer carbon monoxide monitor. A reading of 10ppm and above was used to verify that they were a regular smoker. Resting heart rate was also measured at this point. Research has shown that after 11-15 hours of smoking abstinence, average resting heart rate (HR) drops approximately 8.5 beats per minute in regular smokers (Perkins et al. 1989). As exercise intensity calculations are based on resting heart rate, smokers' heart rates were taken prior to abstinence, as this is more indicative of their normal 
resting heart rate. Participants when joining the study were only told that the study was investigating attitudes to exercise in smokers, this was reflected in the information sheet and the consent form. This was intended to draw participants' attention away from the real aim of this investigation.

Participants then completed a modified version of the credibility scale (Borkovec and Nau 1972). The scale was adapted for use in this study, in consultation with health psychology and smoking cessation experts, and was piloted in a group of smokers. The scale consists of 12 questions related to expectation in the success of four different smoking cessation techniques (hypnotherapy, nicotine patches, meditation and exercise). The three exercise questions were: 'How logical do you consider this approach to smoking cessation to be?' 'How certain are you that this method will be successful in reducing your desire to smoke and withdrawal symptoms?' and 'With what degree of confidence would you recommend this approach to a friend who is trying to stop smoking?' All 12 questions were answered by all participants and were rated on a 10 point scale, $0=$ not at all to $10=$ completely. The alpha value for this scale $=.71$. This reflects an acceptable level of reliability (Kline, 1993). Average time between initial screening (time 1) and the experimental procedure (time 2) was one month.

At the beginning of the experimental procedure (time 2) after temporary overnight smoking abstinence (12-15 hours), carbon monoxide $(\mathrm{CO})$ and resting heart rate measurement was repeated. A reading less than $10 \mathrm{ppm}$ was taken as evidence of smoking abstinence (as used in previous research; Daniel et al. 2006; Ussher et al. 2001). Participants were then randomly allocated to one of the three experimental 
conditions using an SPSS based randomisation procedure. These conditions consisted of a positive, an ambiguous and a negative expectation group. In the positive condition, participants read a prepared statement indicating that exercise is beneficial in terms of withdrawal symptom reduction. In the ambiguous condition participants read two paragraphs of text, one indicating that there is research evidence pointing to the positive effect of exercise on smoking withdrawal and a paragraph stating that other research had found no effect of exercise on withdrawal symptoms. To control for any order effects two versions of the ambiguous statement were used: one presenting the positive paragraph first and vice versa. All statements were piloted in a group of smokers to ensure these statements were understood by smokers. The negative group read a paragraph that stated no effect of exercise on withdrawal had been found by research.

Next, respondents completed the Fagerström test for nicotine dependence (Heatherton et al. 1991), the seven-day physical activity recall questionnaire (Blair et al. 1985), a stages of change for physical activity measure (Marcus et al. 1992), the expectancy measure a second time and two brief questions on desire and intention to quit smoking (Daniel et al. 2006). At this point participants were fitted with a Polar heart rate monitor but they were unable to see the watch interface. During the procedure participants' heart rates were monitored. This allowed for continuous heart rate monitoring at five-second intervals.

Participants then completed the Mood and Physical Symptoms Scale (MPSS) (West and Russell 1985). The MPSS contained the following items: (1) Strength of desire to 
smoke, (2) irritability, (3) depression, (4) tension, (5) restlessness, (6) difficulty concentrating, and (7) stress. Each item was rated on a 7 point scale, ranging from $1=$ not at all, $4=$ somewhat, to $7=$ extremely. In addition, a further item was used to assess desire for a cigarette, 'I have a desire for a cigarette right now' (Tiffany and Drobes 1991), and was rated on a 7 point scale, ranging from 1 = strongly disagree, 4 $=$ neutral, to $7=$ strongly agree. All scales were presented to participants via laminated cards. The MPSS was administered at 10, $5 \& 0$ minutes pre-exercise (these ratings were averaged in order to obtain stable baseline), at 5 and 10 minutes (during exercise) and at 15 and 20 minutes (post-exercise). Social interaction between participants and experimenter was kept to a minimum.

For the exercise component of the procedure a 'Cateye Ergociser' stationary ergometer was used, the seat height was adjustable as was the physical load. Participants were required to exercise for 10 minutes in their moderate intensity range, between 40-60\% of their Heart Rate Reserve (HRR), using the Karvonen method (Karvonen and Vuorimaa 1988). Each individual completed a warm up period lasting one to two minutes (this was not part of the 10 minute experimental period). At 5 minutes into exercise they rated their mood via the MPSS and then repeated at 10 minutes. Participants then dismounted the exercise bike and sat down. At 15 minutes (5 minutes post exercise) participants completed the MPSS again. They then sat quietly for a further 5 minutes and completed the MPSS a final time (20 minutes postexercise). Following completion of the study all participants were debriefed and paid the sum of $£ 10.00$ for their participation.

Ratings of MPSS were averaged across the first three readings, during the two exercise and over the two post-exercise phases of the study, and these data were 
analysed using analysis of covariance with the intervention and post-intervention ratings as the dependent variables, the three expectation conditions as between-subject effects, and pre-exercise baseline ratings as covariates.

\section{RESULTS}

The characteristics of the three groups are presented in Table 1. There were no significant differences between the three groups on baseline characteristics (ANOVA) including baseline ratings of strength of desire to smoke and desire for a cigarette (contact lead author if full means and SD required for MPSS items). Pre-abstinence $\mathrm{CO}$ was significantly greater than $\mathrm{CO}$ after overnight abstinence, $t_{(43)}=17.94, \mathrm{P}<.001$, in all participants. All participants had a CO rating below 10 ppm after 12-15 hours of overnight abstinence. Resting HR pre-abstinence was significantly higher than resting HR during abstinence, $t_{(43)}=2.29, \mathrm{P}<.05$, in all participants.

\section{Table 1 about here}

Figure 1 illustrates average scores on the credibility scale before and after expectation was manipulated. No significant differences in baseline expectation of exercise effects were found when comparing expectation scores at baseline between groups. For the positive group, ratings (1-10) were significantly lower at baseline compared with ratings at time $2, t_{(14)}=-3.10, \mathrm{P}<.01$, effect size, $d=.83$. For the ambiguous group scores at time 1 and time 2 were not significantly different $t_{(14)}=0.30, \mathrm{P}=.70$, effect size, $d=.04$. Ratings in the negative group were significantly lower at time 2 compared with baseline ratings $t_{(14)}=2.20, \mathrm{P}<.05$, effect size, $d=.20$. This indicates that the expectation manipulation had been successful. 
No significant group main effects were found for any item of the MPSS, including desire for a cigarette and desire to smoke. The manipulation had no effect on exercise induced reductions in symptoms. As such, further analysis based on group divisions was unnecessary. In order to determine if withdrawal symptoms and desire to smoke were reduced during and after exercise, paired sample t-tests were conducted to determine if ratings were significantly different for each symptom compared with baseline. Effect sizes were also calculated. These are outlined in Table 2 (data reported in Table 2 consist of mean rating score across 3 baseline recordings, 2 during exercise and 2 post-exercise). All smoking withdrawal symptoms and desire for a cigarette were significantly lower than baseline ratings after exercise. During exercise only restlessness and difficulty concentrating were non-significant although both were significantly lower than baseline ratings post-exercise. Size of effect for each item ranged from what is commonly considered moderate to large effect sizes.

\section{Insert Table 2 about here}

\section{DISCUSSION}

Expectation of the effects of exercise on smoking withdrawal symptoms were successfully manipulated, both reducing and increasing expectation of exercise effects on withdrawal prior to taking part in exercise. Groups were not found to differentially report reductions in withdrawal symptoms and desire to smoke during and following ten minutes of moderate exercise. By making information about exercise effects on withdrawal symptoms and desire to smoke available directly before making judgements on exercise effects on withdrawal, it was possible to manipulate these 
expectations. In the positive group, expectation of the beneficial effects of exercise on withdrawal was increased, and in the negative group expectation decreased. Expectation scores remained unchanged in the neutral group. Regardless of the expectations of participants, withdrawal symptoms were reduced during and following exercise. This suggests that expectation as a mechanism by which exercise reduces smoking withdrawal symptoms is unlikely although inclusion of a passive control condition in future research would lend further support to this possibility.

Thus ten minutes of moderate intensity exercise was found to be effective at reducing acute smoking withdrawal symptoms and desire to smoke in all participants, regardless of participant expectation. In terms of other possible mechanisms responsible for this effect it has been suggested that exercise may produce increases in affect and energy which in turn reduce negative withdrawal symptoms, in line with self-regulation theory (Thayer et al. 1993). This requires further investigation.

As the majority of participants were university students, they were generally younger, had smoked for less time and were less chronically addicted to smoking than the average UK smoker. However, this does not necessarily restrict the generalizability of the findings. A possible limitation of this research was that expectation of exercise effects on withdrawal may have changed since baseline ratings were recorded. As second ratings of expectation were only recorded after the expectation manipulation, it is not possible to discern how stable expectation of exercise effects on withdrawal was across the time between recruitment and testing. Due to the limited time and the possibility of alerting participants to the aims of the research it was not methodologically feasible to administer the credibility measure more than once during 
the testing procedure. Future research should consider measuring credibility scores on more than one occasion prior to testing, and possibly after exercise has been completed. Also, as the expectation measure used within this research was adapted from another scale, future research should attempt to validate the use of this measure in measuring expectation of exercise affects on withdrawal.

In summary, the present findings suggest that expectation of the effects of exercise on withdrawal and desire to smoke does not influence actual reductions in withdrawal symptoms and desire to smoke. 


\section{REFERENCES}

Berger B, Owen D, Motl R, Parrott A (1998) Relationship between expectancy of psychological benefits and mood alteration in joggers. International Journal of Sport Psychology 29:16

Blair SN, Haskell WL, Paffenbarger RS, Vranizan KM, Farquhar JW, Wood PD (1985) Assessment of habitual physical activity by a seven-day recall in a community survey and controlled experiments. American Journal of Epidemiology 122:794-804

Borkovec TD, Nau, SD (1972) Credibility of analogue therapy rationales. Journal of Behaviour Therapy and Experimental Psychiatry 3:257-60

Daniel JZ, Cropley M, Ussher M, West R (2004) Acute effects of a short bout of moderate versus light intensity exercise versus inactivity on tobacco withdrawal symptoms in sedentary smokers. Psychopharmacology 174:320-26

Daniel JZ, Cropley M, Fife-Schaw C (2006) Exercise and smoking withdrawal symptoms: acute effects of 10 minutes of moderate exercise versus 10 minutes of a distraction task. Addiction 101:1187-92

Desharnais R, Jobin J, Cote C, Levesque L, Godin G (1993) Aerobic exercise and the placebo effect: A controlled study. Psychosomatic Medicine 55:149-54

Franklin BA (2000) American College of Sports Medicine Guidelines for Exercise Testing and Prescription. Philadelphia: Lippincott, Williams \& Wilkins 
Heatherton TF, Kozlowski LT, Frecker RC, Fagerström KO (1991) The Fagerström Test for Nicotine Dependence: a revision of the Fagerström Tolerance Questionnaire. British Journal of Addiction 86:1119-27

Karvonen J, Vuorimaa T (1988) Heart rate and exercise intensity during sports activities: Practical application. Sports Medicine 5:303-12

Kline, P (1993). The handbook of psychological testing. London: Routledge.

Marcus B, Rossi R, Selby V, Niaura R, Abrahams D (1992) Self-efficacy and the stages of exercise behaviour change. Health Psychology 11:60-6

O'Halloran P, Murphy G, Webster, K (2002) Measure of beliefs about improvements in mood associated with exercise. Psychological Reports 90:834-40

Perkins K, Epstein L, Stiller R, Marks B, Jacob R (1989) Chronic and acute tolerance to the heart rate effects of nicotine. Psychopharmacology 97:534

Pierce TW, Madden DJ, Siegel WC, Blumenthal JA (1993) Effects of aerobic exercise on cognitive and psychological functioning in patients with mild hypertension. Health Psychology 12:291

Salmon, P (2001) Effects of physical exercise on anxiety, depression, and sensitivity to stress: a unifying theory. Clinical Psychology Review 21:33-61

Schwarz N (2002) Situated cognition and the wisdom of feelings: Cognitive tuning. In: BL Feldman, P Salovey (eds) The wisdom in feelings., New York: Guilford Press, $1014-1026$ 
Taylor A, Katomeri M, Ussher, M (2005) Acute effects of self-paced working on urges to smoke during temporary smoking abstinence. Psychopharmacology 181:1-7

Taylor A. H, Ussher M, Faulkner, G (2007) The acute effects of exercise on cigarette cravings, withdrawal symptoms, affect and smoking behaviour: A systematic review. Addiction 102: 534-543

Thayer R, Peters, D, Takahaski, P, Birkhead-Flight A (1993) Mood and behaviour following moderate exercise. Personality and Individual Differences 14:97-104

Thomas SG, Reading J, Shepard R (1992) Revision of the Physical Activity (PAR-Q) Questionnaire. Canadian Journal of Sport Sciences 14:338-45

Tiffany S, Drobes D (1991) The development and initial validation of a questionnaire on smoking urges. British Journal of Addiction 86:1467-76

Ussher M, Nunziata P, Cropley M, West R (2001) Effect of a short bout of exercise on tobacco withdrawal symptoms and desire to smoke. Psychopharmacology 158:6672

West R, Russell M (1985) Pre-abstinence smoke intake and smoking motivation as predictors of cigarette withdrawal symptoms. Psychopharmacology 87:407-15

Williams JMG, Watts FN, MacLeod C, Mathews, A (1997) Cognitive psychology and emotional disorders. Chichester: Wiley 
Wilson T, Lisle D, Kraft D, Wetzel C (1989) Preferences as expectation driven inferences: effects of affective expectations on affective experience. Journal of Personality and Social Psychology 56:519-30

Youngstedt S, Dishman R, Cureton K, Peacock L (1993) Does body temperature mediate anxiolytic effects of acute exercise? Journal of Applied Physiology 74:825-83 
Table 1: Mean (SD) values for participant characteristics 


\begin{tabular}{|c|c|c|c|c|}
\hline & Positive & Ambiguous & Negative & $\mathrm{p}$-value \\
\hline Age & $24.1(5.5)$ & $24.1(5.5)$ & $23.9(3.9)$ & ns \\
\hline BMI & $23.5(4.2)$ & $25.5(3.3)$ & $26.1(4.5)$ & ns \\
\hline Years Smoked & $7.75(4.8)$ & $7.7(4.8)$ & $6.7(2.5)$ & ns \\
\hline Cigarettes per day & $14(3.7)$ & $16(5.7)$ & $13.2(3.1)$ & ns \\
\hline FTND & $4.3(1.8)$ & $4.1(1.9)$ & $4.1(2.1)$ & ns \\
\hline ECO - time 1 & $26.2(9.1)$ & $28.6(10.9)$ & $27.8(9.1)$ & ns \\
\hline ECO - time 2 & $5.3(3.1)$ & $5.9(2.8)$ & $4.1(2.7)$ & ns \\
\hline Pre-abstinence RHR & $74.6(2.9)$ & $74(3.9)$ & $77.7(1.4)$ & ns \\
\hline Post-abstinence RHR & $73.1(1.6)$ & $72.6(2.7)$ & $75.2(1.5)$ & ns \\
\hline Hours abstinence & $13.2(1.1)$ & $12.5(1.4)$ & $13.2(1.1)$ & ns \\
\hline Motivation to quit (0-6) & $1.8(1.1)$ & $1.8(1.5)$ & $2.4(1.2)$ & ns \\
\hline $\begin{array}{l}\text { Pre-exercise ratings of Strength } \\
\text { of desire to smoke (1-7) }\end{array}$ & $4.4(1.3)$ & $4.4(1.9)$ & $4.3(1.8)$ & ns \\
\hline $\begin{array}{l}\text { Pre-exercise ratings of Desire for } \\
\text { a cigarette (1-7) }\end{array}$ & $4.8(1.6)$ & $4.7(1.9)$ & $4.2(2.1)$ & ns \\
\hline FTND = Fagerström Test for Nic & e Depend & $\mathrm{e}, \mathrm{ECO}=\mathrm{e}$ & ed carbor & noxide, \\
\hline
\end{tabular}


Figure 1: Average rating on the credibility scale taken at Time 1 and Time 2 




Time 1

Time 2 
Table 2: Mean (SD) values for withdrawal symptoms and desire to smoke 
MPSS items

Baseline

During

(pre-exercise)
Exercise
$2.8(1.7)^{* * *}$

$3.1(1.4)$

$2.1(1.1)$

$2.9(1.3)$

$3.0(1.4)$

$3.0(1.7)$

$3.0(1.5)$

4.5 (1.6)

$2.6(1.4)^{* *}$

$1.7(1.3)^{*}$

$2.3(1.1)^{* *}$

$2.5(1.4)$

$2.8(1.3)$

$2.4(1.4)^{*}$

$2.7(1.6)^{* * *}$
0.8

0.8

$2.9(1.5)^{* * *}$

$2.1(.9)^{* *}$

0.9

0.5

$1.6(.8)^{*}$

0.5

0.5

$2.0(.9)^{* *}$

0.4

ns

$2.1(1.1)^{* *}$

0.7

Desire for a cigarette

$*$ = significantly different from baseline, $\mathrm{p}<.05 ; * *=$ significantly different from baseline, $\mathrm{p}<.01$;

*** = significantly different from baseline, $\mathrm{p}<.001$ 